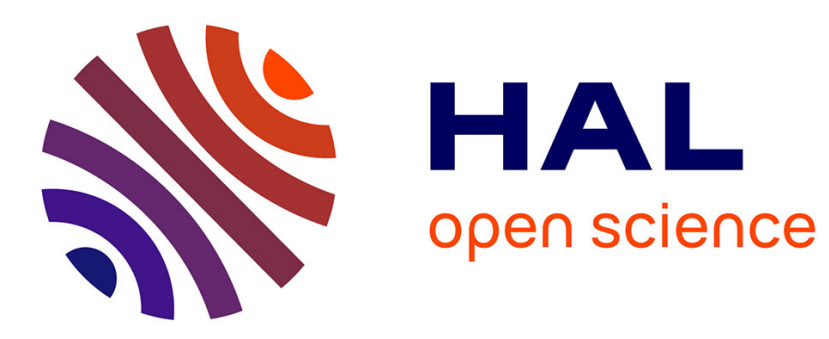

\title{
Mixing Hough and Color Histogram Models for Accurate Real-Time Object Tracking
}

Antoine Tran, Antoine Manzanera

\section{To cite this version:}

Antoine Tran, Antoine Manzanera. Mixing Hough and Color Histogram Models for Accurate RealTime Object Tracking. Computer Analysis of Images and Patterns (CAIP), 2017, Ystad, Sweden. hal-01671906

\section{HAL Id: hal-01671906 https://hal.science/hal-01671906}

Submitted on 22 Dec 2017

HAL is a multi-disciplinary open access archive for the deposit and dissemination of scientific research documents, whether they are published or not. The documents may come from teaching and research institutions in France or abroad, or from public or private research centers.
L'archive ouverte pluridisciplinaire HAL, est destinée au dépôt et à la diffusion de documents scientifiques de niveau recherche, publiés ou non, émanant des établissements d'enseignement et de recherche français ou étrangers, des laboratoires publics ou privés. 


\title{
Mixing Hough and Color Histogram Models for Accurate Real-Time Object Tracking
}

\author{
Antoine Tran and Antoine Manzanera \\ U2IS, ENSTA ParisTech \\ Université de Paris-Saclay \\ 828, Bd des Maréchaux \\ 91762 Palaiseau CEDEX - France \\ antoine. [tran, manzanera] @ensta-paristech.fr
}

\begin{abstract}
This paper presents a new object tracking algorithm, which does not rely on offline supervised learning. We propose a very fast and accurate tracker, exclusively based on two complementary low-level features: gradient-based and color-based features. On the first hand, we compute a Generalized Hough Transform, indexed by gradient orientation. On the second hand, a RGB color histogram is used as a global rotation-invariant model. These two parts are processed independently, then merged to estimate the object position. Then, two confidence maps are generated and combined to estimate the object size. Experiments made on VOT2014 and VOT2015 datasets show that our tracker is competitive among all competitors (in accuracy and robustness, ranked in the top 10 and top 15 respectively), and is one of the few trackers running at more than $100 \mathrm{fps}$ on a laptop machine, with one thread. Thanks to its low memory footprint, it can also run on embedded systems.
\end{abstract}

\section{Introduction}

Object tracking is a very popular task in computer vision. Basically, the goal is to accurately localize one defined object (the target) in a video. Among difficulties, we can cite object deformation, motion change, rotation, scaling or those linked to the context (illumination change, occlusion, camouflage, camera motion). Applications such as human-computer interaction or augmented reality require reactive algorithms, so the computational cost may be a critical issue.

For efficiency, our tracker is based on very light methods, and combines lowlevel shape and color features. It is a model-free tracker, meaning that the offline training is done only at the first frame of the sequence. The gradient orientation is computed and used as an index of a Generalized Hough Transform (GHT) [2]. A RGB histogram is used to represent the color aspect of the target object, and distinguish it from the background. These two parts are processed independently, and merged to finally estimate the object location. They are complementary: the original GHT is robust to illumination, but weak against scale and rotation, unlike the color histogram model. 
By testing and evaluating our tracker on VOT2014 and VOT2015 [15, 16], we show that this combination leads to high performance in terms of accuracy and robustness. Moreover, by associating low-level features and light algorithms, our tracker can run at more than $100 \mathrm{fps}$ on a laptop machine, without explicit multithreading. It is one of the fastest among all competitors, while being ranked second among real-time trackers in terms of accuracy and robustness criteria from VOT2015. Its lightness also makes it suitable to embedded systems.

This paper is organized that way: after a short state-of-the-art of object tracking, we will explain our method. Finally, we will show some results obtained in the academic datasets.

\section{Related Work}

Given a sequence, object tracking consists in estimating the state of one target object at each frame. This state can be its center, its bounding box or its silhouette. Many works have addressed the tracking problem, and we refer to Yilmaz' survey for a coverage of the task [25].

Structurally, our tracker belongs to the class of trackers combining different methods $[1,3,9,18,24]$. Among recent works, STAPLE [3] is related to our technique: it combines correlation and color histogram model to provide an effective and fast tracker. As we are using the GHT in its original form, our templatepart tracker is simpler and lighter than Bertinetto's, as it only requires gradient computation (instead of HOG features [5]). Duffner's PixelTrack [9] is also close to our tracker: it combines a GHT with a foreground/background color model into a very fast tracking algorithm (above $100 \mathrm{fps}$ ).

The VOT committee annually proposes a dataset to evaluate and rank trackers [14-16]. In this challenge, the most accurate trackers are based on Convolutional Neural Network $[19,20]$ and correlation filters $[3,7]$. If we focus on the fastest algorithms of VOT15 challenge, Vojir's tracker [23] based on the Meanshift [4] proposes decent accuracy, with a speed far beyond real-time. Maresca [18] proposes a fast tracker based on estimation of object motion, obtained by the combination of several light trackers.

Our proposed tracker is based on very low-level features and lightweight operations: color histogram and GHT. Color histogram is popular in object tracking $[4,21,23]$, since it is fast and robust to scale and in-plane rotation changes. The GHT is an extension of the Hough Transform [8], used to detect arbitrary shapes. It consists in considering some elements of this shape (pixels, patches) and, according to their local appearances, make them vote for potential positions of the shape center. The estimated center is then determined by the location that has accumulated the highest number of votes. The GHT is robust to illumination changes and to camouflage issue, compensating some weaknesses of the color-based model. In tracking context, the main issue of the GHT is the robustness to scale and rotation changes. However, several authors proposed Hough-based trackers $[9-11,17]$ by modifying the original GHT, or using complementary methods. Amongst Hough-based trackers, only Hua [13] outperforms 
our algorithm, but is 100 times slower. However, his algorithm essentially relies on a HOG-based detector, which estimates several candidates locations, and the Hough Transform is only used to discard wrong candidates. PixelTrack [9] is at a same order of magnitude in speed, but much less accurate and robust.

In the last VOT challenges $[14,15]$, few trackers belong to the category of realtime trackers (21 competitors among 132), with diverse performances in accuracy and robustness. In this category, our method is one of the fastest (more than $100 \mathrm{fps}$ ), but also one of the most accurate and robust (only beaten by [23]). Compared to slower tracker, ours is still competitive: ranked in the top 15 for both criteria in VOT2015 [15] and ranked in the top 10 in VOT2014 [16].

Our state representation is based on a bounding box. Given a frame $t$, the aim is to estimate the bounding box $B_{t}=\left(c_{t}, w_{t}, h_{t}\right)$ of an object $\mathcal{O}$, where each parameter is respectively: bounding box's center, width and height.

\section{Our contribution}

First, we explain how to initialize our tracker. Second, we deal with state estimation. As position is estimated before scale, we will explain these two steps in different sections. Third, we explain the model updating process.

\section{$3.1 \quad$ Initialization}

Our tracker is initialized at the first frame $\mathbf{I}_{0}$, with a manually set bounding box $B_{0}$. Let $\mathbf{I}(B)$ be the restriction of an image $\mathbf{I}$ to any subset of pixels $B$.

First, the target RGB histogram $H_{0}$ of $\mathbf{I}_{0}\left(B_{0}\right)$, with $n_{c} \times n_{c} \times n_{c}$ bins, is generated $\left(n_{c}=12\right) . H_{t}$ will denote the target RGB histogram at the frame $t$.

Second, for the geometrical model, let $\mathbf{M}_{0}$ and $\boldsymbol{\Phi}_{0}$ be the gradient magnitude and orientation of $\mathbf{I}_{0}\left(B_{0}\right)$. The goal is to initialize the $R$-Table $R$ (which will be updated over the time), indexed by $n_{o}=16$ orientations. It consists in considering all pixels $p \in \mathbf{I}_{0}\left(B_{0}\right)$, for which $\mathbf{M}_{0}(p)>\epsilon_{M}\left(\epsilon_{M}=70.0\right)$ and whose quantified gradient orientation is $\theta_{p}$, then to store in $R\left(\theta_{p}\right)$ the couple $\left(\vec{u}=\overrightarrow{p c_{0}}, \omega_{\vec{u}}\right)$, which is the displacement from $p$ to the bounding box center $c_{0}$, and $\omega_{\vec{u}}=1.0$ a default weight value.

\subsection{Estimation of position}

Given an image $\mathbf{I}_{t}$ from a sequence, our tracker first estimates the object center, then its scale. We will explain these two steps independently.

On the one hand, we perform a basic GHT. As during initialization, it requires to evaluate gradient image from $\mathbf{I}_{t}$, keep pixels $p$ whose gradient magnitude is above $\epsilon_{M}$, and quantify its orientation as $\theta_{p}$. Then, for each couple $\left(\vec{u}, \omega_{\vec{u}}\right) \in$ $R\left(\theta_{p}\right), p$ votes for all displacements $p+\vec{u}$ with the weight $\omega_{\vec{u}}$. More formally:

$$
\mathbf{H T}_{t}(p)=\sum_{q} \sum_{(\vec{u}, \omega) \in R\left(\theta_{q}\right)} \omega \cdot \delta(p, q+\vec{u})
$$




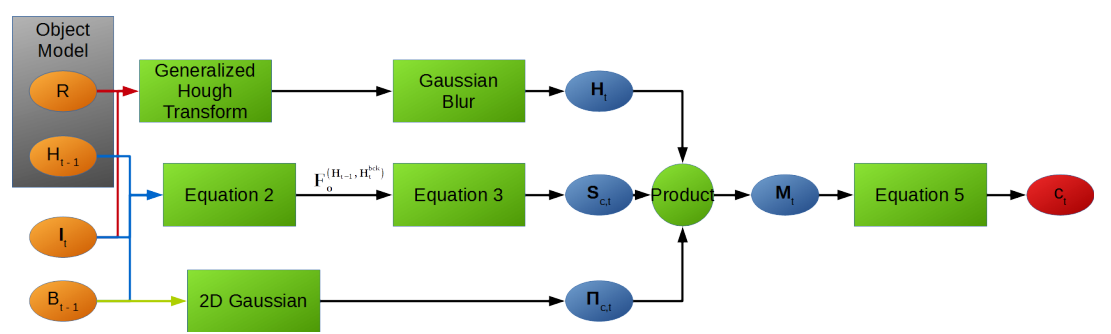

Fig. 1: Position tracking diagram.

with $\delta$ the Kronecker function. Finally, when all pixels have voted, the created map (the Hough Transform) $\mathbf{H T}_{t}$ emphasizes the most probable locations of the object center, with respect to the geometrical model. The GHT is performed under its simplest form, unlike [9] who indexes their R-Table using gradient and color features and $[11,17]$ who use machine learning classifiers.

We then blur $\mathbf{H T}_{t}(3 \times 3$ discrete Gaussian filter $)$, in order to add robustness to deformation. The GHT is intrinsically robust to illumination change (conservation of the orientation), but not to scaling (pixels votes spread away from the center) nor to rotation (pixels vote according to the wrong list in the R-Table).

On the other hand, to exploit the color model, we define foreground and background areas. We use the color model histogram $H_{t-1}$, and build a background histogram $H_{t}^{b c k}$. These features have the advantage to compensate for the GHT weaknesses, and vice-versa. Given the last estimated bounding box $B_{t-1}$, let $S_{t}$ be the background area, defined by all pixels inside the bounding box of center $c_{t-1}$, and dimension $\left(\alpha \cdot w_{t-1}, \alpha \cdot h_{t-1}\right)(\alpha=2.0)$, excluding $B_{t-1}$. From $S_{t}$, we build $H_{t}^{b c k}$. Then, for every pixel $p \in\left(B_{t-1} \cup S_{t}\right)$, let $q_{t}^{p}$ be its quantified color in $\mathbf{I}_{t}$. As proposed in [21], we define the foregroundness with respect to the object $\mathcal{O}$, knowing the object color histogram $H_{t-1}$ and the background color model:

$$
\mathbf{F}_{\mathcal{O}}^{\left(H_{t-1}, H_{t}^{b c k}\right)}(p)= \begin{cases}\frac{H_{t-1}\left(q_{t}^{p}\right)}{H_{t-1}\left(q_{t}^{p}\right)+H_{t}^{b c k}\left(q_{t}^{p}\right)} & \text { if } p \in\left(B_{t-1} \cup S_{t}\right) \\ 0 & \text { otherwise }\end{cases}
$$

$\mathbf{F}_{\mathcal{O}}^{\left(H_{t-1}, H_{t}^{b c k}\right)}(p)$ indicates how probably $p$ belongs to the target. Unlike [21], we do not combine Eq. 2 with a distractor-aware model, as we aim to remain as simple as possible. Compared to PixelTrack [9], this method only needs a model histogram and no prior information about background. Then, given a bounding box $B$ of size $\left(w_{t-1}, h_{t-1}\right)$ inside $\left(B_{t-1} \cup S_{t}\right)$, let $\mathbf{S}_{c, t}(B)$ be the normalized score evaluating whether the target is inside $B$ :

$$
\mathbf{S}_{c, t}(B)=\frac{\sum_{p \in B} F_{\mathcal{O}}^{\left(H_{t-1}, H_{t}^{b c k}\right)}(p)}{w_{t-1} \cdot h_{t-1}}
$$

This formulation is simpler than Possegger's [21], who proposed a method to discard distractors. We also use a prediction map, that indicates the likelihood 
to find $c_{t}$ at $x$, given $B_{t-1}$ :

$$
\boldsymbol{\Pi}_{t}^{B_{t-1}}(x)=\exp \left(\frac{-\left(x-c_{t-1}\right)^{2}}{2 \cdot \min \left(w_{t-1}, h_{t-1}\right)^{2}}\right)
$$

Finally, for all pixels $x$, we define $\mathbf{M}_{t}$ such as $\mathbf{M}_{t}(x)=\mathbf{H T}_{t}(x) \cdot \mathbf{S}_{c, t}\left(B_{x}\right)$. $\mathbf{\Pi}_{t}^{B_{t-1}}(x)$ with $B_{x}$ the rectangle centered in $x$ of size $\left(w_{t-1}, h_{t-1}\right)$. From $\mathbf{M}_{t}$, we finally estimate the object position $c_{t}$ as follows:

$$
c_{t}= \begin{cases}\underset{x}{\operatorname{argmax}}\left(\mathbf{M}_{t}(x)\right) & \text { if } \max _{x}\left(\mathbf{M}_{t}(x)\right) \neq 0 \\ c_{t-1}+\overrightarrow{c_{t-2} c_{t-1}} & \text { otherwise }\end{cases}
$$

The second case of Eq. 5 assumes that, when the support of $\mathbf{H T}_{t}$ and $\mathbf{S}_{c, t}$ are disjoint, the target is translating with a vector $\overrightarrow{c_{t-2} c_{t-1}}$. We choose a pixel-wise multiplication to merge our two trackers, unlike STAPLE [3] who used a weighted average. In that way, we do not have to deal with the difference of magnitude of $\mathbf{H T}_{t}$ and $\mathbf{S}_{c, t}$, and to adjust a weight. We also differ from PixelTrack [9], where the final position is obtained by linear combination of the centers estimated by the GHT on one hand and the color segmentation map on the other hand. Fig. 1 describes all steps for our position estimation.

\subsection{Estimation of scale}

The second step consists in estimating object scale.

On the one hand, from the GHT, let us define the backprojection map $\mathbf{B P} \mathbf{P}_{t}$, for all pixels $\mathrm{p}$ fulfilling conditions to be stored in the R-Table:

$$
\mathbf{B P}_{t}(p)=\frac{\sum_{(\vec{u}, \omega) \in R\left(\theta_{p}\right)} \mathbf{M}_{t}(p+\vec{u})}{\left|R\left(\theta_{p}\right)\right|}
$$

with $\left|R\left(\theta_{p}\right)\right|$ the cardinality of $R\left(\theta_{p}\right)$. The approach is similar to Duffner's one [9]. However, Duffner only backprojects the peak of the GHT, while we consider the sum of the voted positions for all pixels. In both cases, the made assumption is that the higher the backprojection is, the more likely it belongs to the target.

On the other hand, we consider $\mathbf{F}_{\mathcal{O}}^{\left(H_{t-1}, H_{t}{ }^{b c k}\right)}$, defined Eq. 2, as a color confidence map. Then, let $\mathbf{B} \mathbf{F}_{t}$ be the final confidence map:

$$
\mathbf{B F}_{t}=0.5 \cdot\left(\mathbf{B P}_{t}+\mathbf{F}_{\mathcal{O}}^{\left(H_{t-1}, H_{t}{ }^{b c k}\right)}\right)
$$

Fig. 2 illustrates these maps. Then, inspired by Posseger [21], we consider the set of object pixels $\mathrm{OP}_{t}=\left\{p \mid \mathbf{B F}_{t}(p)>0.5\right\} \cup R_{t}$, with $R_{t}$ a safe foreground area defined as the rectangle centered on $c_{t}$, of size $\left(\beta \cdot w_{t-1}, \beta \cdot h_{t-1}\right)(\beta=0.20)$. From $\mathrm{OP}_{t}$, we only retain the connected component that contains $c_{t}$, to discard isolated pixels that could generate scale overestimation. Finally, we estimate a potential bounding box by computing the bounding box $\bar{B}_{t}$ of this connected 


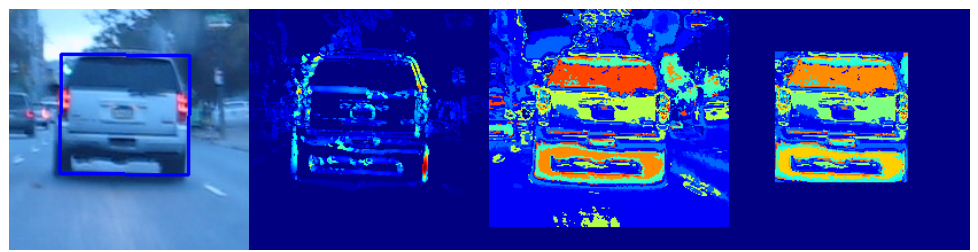
(a) $\mathbf{I}_{t}$
(b) $\mathbf{B} \mathbf{P}_{t}$
(c) $\mathbf{F}_{\mathcal{O}}^{\left(H_{t-1}, H_{t}^{b c k}\right)}$
(d) $\mathbf{B} \mathbf{F}_{t}$

Fig. 2: Cropped frame from car1 from VOT2015, with the ground truth in blue. Backprojection map $\mathbf{B} \mathbf{P}_{t}$ and pixel color likelihood map $\mathbf{F}_{\mathcal{O}}^{\left(H_{t-1}, H_{t}^{b c k}\right)}$ are complementary: while the first one indicates which border pixels are more likely to belong to the target, the second one gives high results for pixels of car's back.

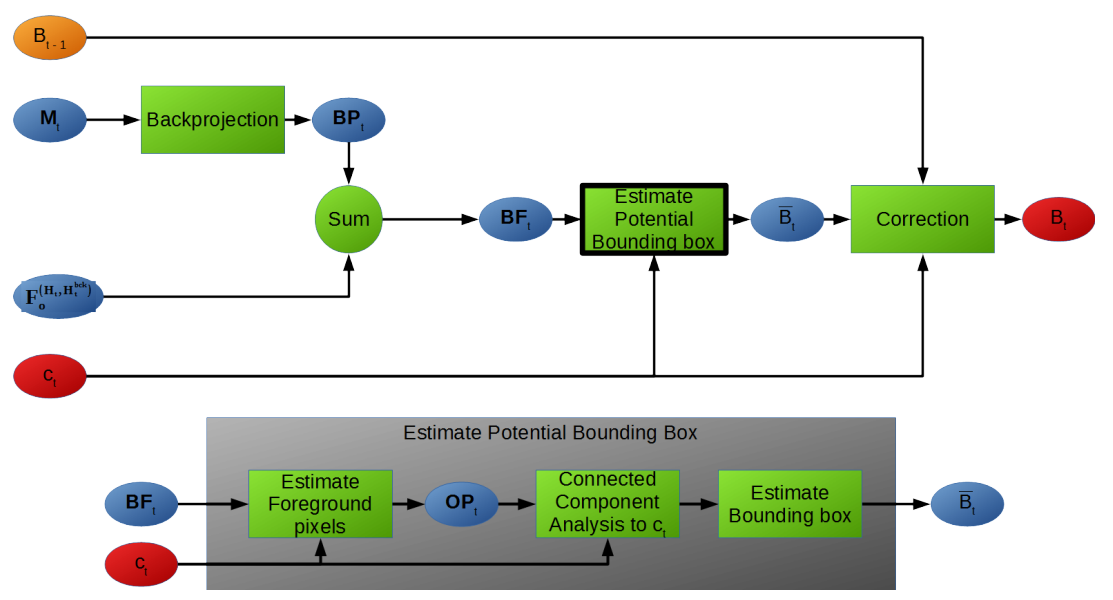

Fig. 3: Scale estimation diagram.

component. Then, we reject bounding box sizes whose relative area variation with respect to $B_{t-1}$ is above $5 \%$. Otherwise, we update object's size using the same aspect ratio, as follows:

$$
X_{t}=\lambda_{t} \cdot X_{t-1}
$$

with $X \in\{w, h\}$ and $\lambda_{t}=\min \left(1.05, \max \left(0.95, \frac{\mathcal{A}\left(\bar{B}_{t}\right)}{\mathcal{A}\left(B_{t-1}\right)}\right)\right)$ ( $\mathcal{A}$ being the area operator). Fig. 3 summarizes scale estimation operations. Finally, to prepare the updating process, let $\mathbf{S G}_{t}$ be the shape and color-based confidence map such that $\mathbf{S G}_{t}(p)=\mathbf{B F}_{t}(p)$ if $p \in B_{t}$, and 0 otherwise.

\subsection{Model update}

The last step consists in updating the model, knowing the estimated bounding box $B_{t}$. To update the color model, let $H_{t}^{o}$ be the color histogram of $\mathbf{I}_{t}\left(B_{t}\right)$ and 


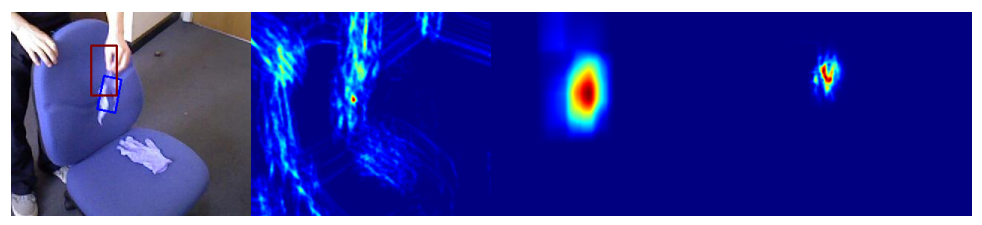
(a)
Cropped
(b) $\mathbf{H T}_{t}$
(c) $\mathbf{S}_{c}, t$
(d) $\mathbf{M}_{t}$ frame (glove)

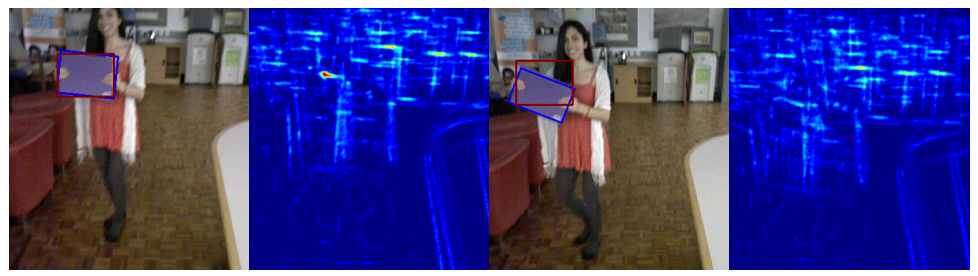
(e) Cropped
frame 1 (book)
(f) $\mathbf{H} \mathbf{T}_{1}$
(g) Cropped
(h) $\mathbf{H T}_{2}$

Fig. 4: The first line illustrates failures due to the transparency of the glove (ground truth in blue, tracker output in red), making its color similar to the chair. The GHT still performs correctly (sharp peak on the second image).

On the second line the GHT fails due to the rotation of the book: the peak disappears from $\mathbf{H T}_{1}$ to $\mathbf{H T}_{2}$.

$\mu_{c}=0.05$ the color updating rate:

$$
H_{t}=\left(1-\mu_{c}\right) \cdot H_{t-1}+\mu_{c} \cdot H_{t}^{o} .
$$

To update the R-Table $R$, we start by reducing all displacement weights:

$$
\forall \theta, \forall\left(\vec{u}, \omega_{\vec{u}}\right) \in R(\theta), \omega \vec{u} \leftarrow\left(1-\mu_{g}\right) \cdot \omega_{\vec{u}}
$$

Then, considering the confidence map $\mathbf{S G}_{t}$, and the object center $c_{t}$, for all pixels $p \in B_{t}$ with gradient orientation $\theta_{p}$, we consider the displacement $\vec{v}=\overrightarrow{p c_{t}}$, with a weight equal to $\mu_{g} \cdot \mathbf{S G}_{t}(p)$. Then, we consider two cases:

- if $\vec{v}$ is in $R\left(\theta_{p}\right)$, we increment its weight by $\mu_{g} \cdot \mathbf{S} \mathbf{G}_{t}(p)\left(\mu_{g}=0.05\right)$, in order to reinforce the most relevant elements of the R-Table

- otherwise we add an entry into $R\left(\theta_{p}\right)$ with the weight $\mu_{g} \cdot \mathbf{S G}_{t}(p)$

Finally, for each index of the R-Table, we keep only the $N_{R}=200$ displacements with the strongest weights, to limit computational and memory cost.

\section{Experiments}

Before dealing with experiments on academic datasets, we will detail our implementation setup. 


\subsection{Implementations details}

Our algorithm is developed using $\mathrm{C}++$ and the OpenCV 2.4.9 library, and tested on a laptop at $2.4 \mathrm{GHz}$, without explicit multithreading. In terms of implementation, iterative pixel access is done using image pointers, and image histogram computation is done using Look Up Table. At frame $t$, our tracker only processes area centered in $c_{t-1}$, and of size two times the last bounding box area. To evaluate the map $\mathbf{S}_{c, t}$, we use integral images. Otherwise, no major algorithmic optimization has been done.

In terms of memory footprint, target's informations, composed of the color histogram $\left(n_{c}{ }^{3}=12^{3}\right.$ floating point numbers $)$, the R-Table $\left(n_{0} \cdot N_{R} \cdot 2 \cdot 4\right.$ integers for displacements and $n_{0} \cdot N_{R} \cdot 2$ floats for weights) and the two last states (4 integers for coordinates and scales), resulting, with our set of parameters, in a memory footprint of about 45 ko. This quantity is independent of target' size. It is however negligible compared to the number of temporary images: 2 8-bit images (gradient orientation and magnitude maps), 1 RGB image (the sub-image in which we are tracking the target) and 5 32-bit images (float) $\left(\mathbf{H T}_{t}, \mathbf{B P}_{t}\right.$,

$\mathbf{S}_{c, t}, \mathbf{F}_{\mathcal{O}}^{\left(H_{t-1}, H_{t}^{b c k}\right)}$ and $\mathbf{B F}_{t}$ ), which depends on object's size (and its associated search window): for a $100 \times 100$ object's size, the footprint will reach 2 Mo. Considering the speed obtained experimentally, we are convinced that our method is suitable embedded systems. The code of our implementation will be made available soon. All parameters have been tuned for the best trade-off between performance and speed on VOT2015 [15]. For experiments case, we will denote as CHT the position tracker only, and CHTs our complete tracker.

\subsection{Results on VOT 2014 and VOT 2015}

Each year, the VOT committee proposes a dataset to test and evaluate trackers. Each frame from each sequence is labeled according to its difficulty (occlusion, camera motion, size, motion or illumination change). Evaluation criteria are:

- Accuracy: based on overlap measure $O\left(G T_{t}, B_{t}\right)=\frac{G T_{t} \cap B_{t}}{G T_{t} \cup B_{t}}$, with $G T_{t}$ the ground truth at the frame $t$

- Robustness: given by the number of failures (frames where $O\left(G T_{t}, B_{t}\right)=0$ )

- Speed: based on a normalized speed (EFO units, see [15])

The VOT committee provides results of all competitors, and a toolkit to evaluate and rank trackers. For all experiments, we use the function report_challenge to get weighted mean rank (based on ranks for all difficulties), pooled rank (based on all sequences), expected overlap, and speed. We also compute ranking with the whole set of results, but only display those of relevant trackers. Results for VOT2014 and VOT2015 are summarized Tab. 1.

VOT2014 [16] is a dataset composed of 25 sequences, and results for 40 trackers are available. We choose to show our results compared to DSST [6] (VOT2014 winner), Hough-based trackers $[9,17,18]$ and real-time trackers $[9,12$, 18,22]. Amongst Hough-based trackers, our method is as well ranked as Matflow, 


\begin{tabular}{|c|c|c|c|c|c|c|c|}
\hline \multicolumn{8}{|c|}{ VOT2014 } \\
\hline Tracker & $\begin{array}{r}\text { Weighted } \\
\text { Accuracy }\end{array}$ & $\begin{array}{l}\text { mean rank } \\
\text { Robustness }\end{array}$ & $\begin{array}{r}\text { Poole } \\
\text { Accuracy }\end{array}$ & $\begin{array}{l}\text { ed rank } \\
\text { Robustness }\end{array}$ & $\begin{array}{c}\text { Expected } \\
\text { overlap }\end{array}$ & $\begin{array}{r}\mathrm{Sp} \\
(\mathrm{EFO})\end{array}$ & $\begin{array}{l}\text { eed } \\
\text { (fps) }\end{array}$ \\
\hline CHTs & 8.83 & 4.33 & 6 & 9 & 0.2960 & 129.77 & 159.21 \\
\hline СHT & 8.42 & 4.08 & 6 & 9 & 0.2916 & 109.75 & 134.65 \\
\hline DSST [6] & 1.83 & 4.33 & 1 & 3 & 0.3693 & 5.80 & 13.07 \\
\hline Matrioska [17] & 9.83 & 12 & 6 & 9 & 0.2671 & 10.20 & 21.88 \\
\hline bdf $[18]$ & 10.67 & 7.50 & 10 & 9 & 0.3097 & 46.82 & 100.45 \\
\hline Matflow $[17,18]$ & 8.67 & 3.17 & 6 & 4 & 0.3120 & 19.08 & 40.94 \\
\hline FoT $[22]$ & 7 & 18.17 & 6 & 22 & 0.2859 & 114.64 & 306.52 \\
\hline PTp [9] & 25.33 & 11.17 & 30 & 9 & 0.2519 & 49.89 & 127.87 \\
\hline $\mathbf{K C F}[12]$ & 2 & 4.67 & 1 & 5 & 0.3641 & 24.23 & 63.42 \\
\hline \multicolumn{8}{|c|}{ VOT2015 } \\
\hline CHTs & 12.67 & 13.67 & 13 & 17 & 0.2606 & 103.89 & 111.22 \\
\hline CHT & 13.83 & 15.67 & 13 & 20 & 0.2615 & 101.91 & 109.10 \\
\hline Staple [3] & 1 & 4.33 & 1 & 5 & 0.345 & & \\
\hline ASMS [23] & 7.50 & 11 & 2 & 13 & 0.2353 & 115.09 & 142.26 \\
\hline bdf $[18]$ & 29.33 & 32 & 27 & 43 & 0.2054 & 200.24 & 78.43 \\
\hline FoT $[22]$ & 19.50 & 42.50 & 16 & 53 & 0.1934 & 143.62 & 177.53 \\
\hline $\operatorname{DSST}[6]$ & 4.0 & 23.67 & 1 & 38 & 0.2707 & 3.29 & 4.47 \\
\hline $\mathrm{DAT}[21]$ & 13.73 & 17.33 & 6 & 20 & 0.2428 & 9.82 & 14.87 \\
\hline $\mathrm{HT}[11]$ & 20 & 28.50 & 13 & 43 & 0.2045 & 0.91 & 0.56 \\
\hline Matflow $[17,18]$ & 22.17 & 27.33 & 23 & 43 & 0.2098 & 81.34 & 31.86 \\
\hline MDNET [20] & 1 & 1.33 & 1 & 1 & 0.3789 & 0.87 & 0.97 \\
\hline sPST [13] & 1.67 & 4.50 & 1 & 5 & 0.3134 & 1.03 & 1.16 \\
\hline
\end{tabular}

Table 1: Results in VOT2014 and VOT2015 for different trackers. Real-time trackers appear in bold.

combining Matrioska [17] and bdf [18], but is 6 times faster (in EFO units). In the real-time trackers category, ours is the second fastest one, beaten by FoT [22] but our method is much more robust. KCF [12] is more accurate and robust than our method, but is slower. Globally, our tracker is well ranked (top 10 among 40 competitors) but is one of the tracker proposing the best trade-off between effectiveness and speed. Surprisingly, our position-only tracker (CHT) and our complete one have similar performance in terms of accuracy and robustness, but the complete one is faster (probably due to object size reduction).

VOT2015 challenge is composed of 60 sequences and results for 62 competitors are available. Compared to MDNET [20], VOT2015 winner based on CNNs, and STAPLE [3] (results from author's website, without speed results) our tracker is less effective, but faster (being lighter, and knowing that the author mentioned $80 \mathrm{fps}$ on a $4.0 \mathrm{GHz} \mathrm{CPU}$, we expect our method to be faster than STAPLE). Compared to STAPLE, our shape-based tracker relies on the GHT, with gradient computation only, while STAPLE requires a more complex algorithm (correlation-based tracker using HOG features). Amongst real-time trackers $[18,22,23]$, our tracker is one of the few above $100 \mathrm{EFO}$, and is only beaten by Vojir's extension of Meanshift [23] in rankings and speed, but with 

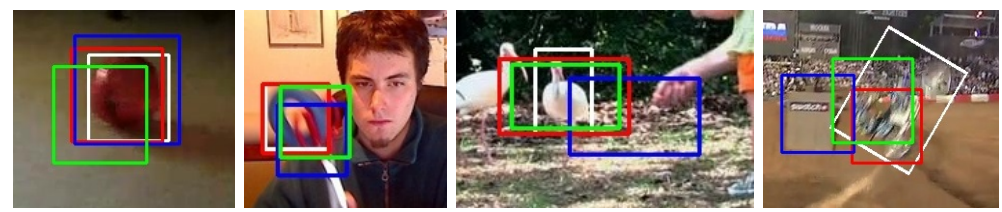

Fig. 5: Results from VOT2014 and 2015.

White bounding boxes are ground truth, red ones are obtained with our tracker. The two first images are cropped from sphere and torus sequences from VOT2014 (PixelTrack in blue, and MatFlow in green).

The two last are cropped from birds2 and motocross sequences from VOT2015 (DAT in blue, and STAPLE in green).

slightly higher expected overlap. Otherwise, we are better ranked than other real-time trackers. We perform better than other Hough-based trackers [11, 17], including Matflow, which was on par with our tracker in VOT2014, but excluding sPST [13], which is 100 times slower and relies on an object detector. Compared to Possegger [21], from which our color part tracker is inspired, we demonstrate the usefulness of the Hough part, since, in weighted mean ranking, our method is slightly more robust. Some results are shown Fig 4 and Fig 5. We also used VOT2015 to see performances of each part of the tracker (Hough and color ones). In both cases, the loss of performance is dramatic, as we can see on Fig. 6, where we show the results of different versions in terms of expected overlap (the higher, the better) and failures (the lower, the better) for several difficulties, both obtained on the set of frames concerned by the difficulties.

\section{Conclusion}

In this paper, we proposed a tracker working without offline training and tracking arbitrary objects. Unlike most state-of-the-art trackers, our method is based on a very low level representation. First, our geometrical model is only based on gradient, through a GHT. Then, an object color histogram is used to generate a map indicating the likeliness of the pixel to belong to the object. These two operations, done independently, are combined to estimate object center. Second, from the two features, we generate two confidence maps, and merge them in order to estimate the object size. The final confidence map is built from the fusion of these two maps and used to update the object geometrical and color models. Our whole tracker relies on computationally efficient operations, and performs tracking task beyond real-time (about $100 \mathrm{fps}$ ) with a low memory footprint. Experiments were done on recent academic dataset $[15,16]$, for which our tracker is ranked in the first third for accuracy and robustness. It is also one of the fastest from VOT2014 and VOT2015 challenges. Thanks to its speed and low memory footprint, our algorithm can be implemented on embedded systems, combined with other trackers to improve accuracy and robustness or with object detection or background subtraction to automatically initialize tracked regions. 

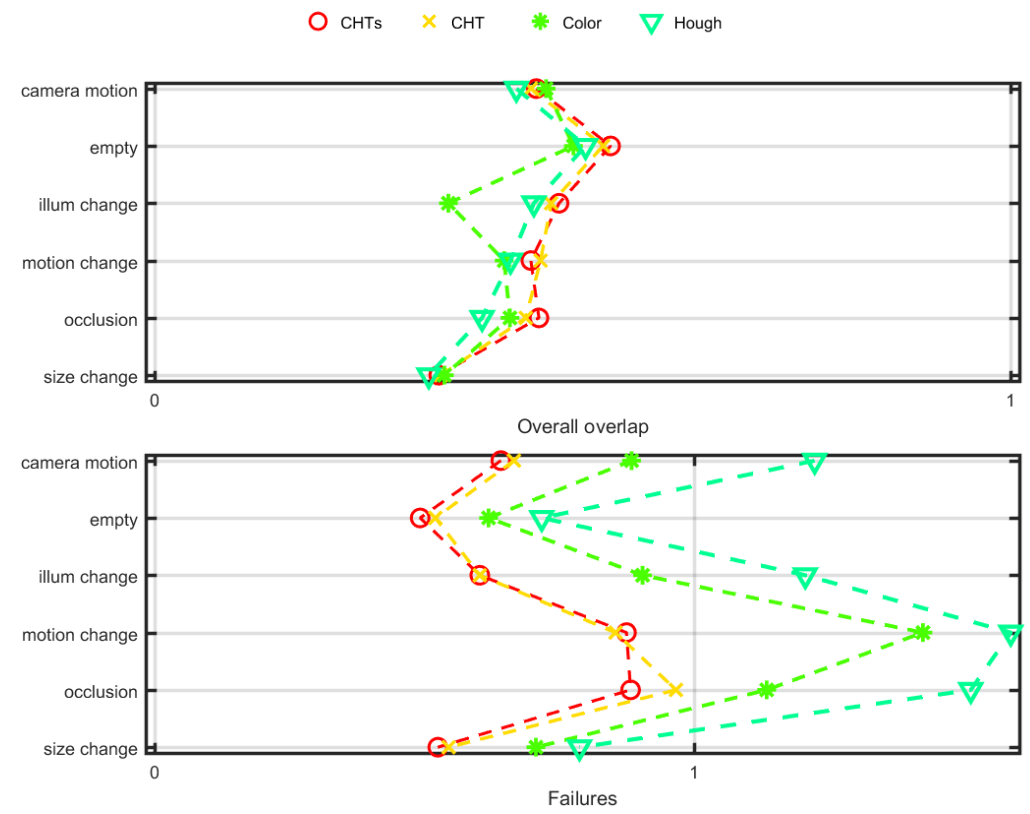

Fig. 6: Expected overlap and number of failures for our complete tracker, and for partial versions (position tracking only, Hough only and Color only)

\section{Acknowledgments}

We gratefully acknowledge financial support from the French Government Defense procurement and technology agency (DGA/MRIS).

\section{References}

1. Babenko, B., Yang, M.H., Belongie, S.: Robust object tracking with online multiple instance learning. IEEE transactions on pattern analysis and machine intelligence 33(8), 1619-1632 (2011)

2. Ballard, D.H.: Generalizing the Hough transform to detect arbitrary shapes. Pattern recognition 13(2), 111-122 (1981)

3. Bertinetto, L., Valmadre, J., Golodetz, S., Miksik, O., Torr, P.: Staple: Complementary learners for real-time tracking. arXiv preprint arXiv:1512.01355 (2015)

4. Comaniciu, D., Ramesh, V., Meer, P.: Real-time tracking of non-rigid objects using mean shift. In: Computer Vision and Pattern Recognition, 2000. Proceedings. IEEE Conference on. vol. 2, pp. 142-149. IEEE (2000)

5. Dalal, N., Triggs, B.: Histograms of oriented gradients for human detection. In: 2005 IEEE Computer Society Conference on Computer Vision and Pattern Recognition (CVPR'05). vol. 1, pp. 886-893. IEEE (2005)

6. Danelljan, M., Häger, G., Khan, F., Felsberg, M.: Accurate scale estimation for robust visual tracking. In: British Machine Vision Conference, Nottingham, September 1-5, 2014. BMVA Press (2014) 
7. Danelljan, M., Robinson, A., Khan, F.S., Felsberg, M.: Beyond correlation filters: Learning continuous convolution operators for visual tracking. In: European Conference on Computer Vision. pp. 472-488. Springer (2016)

8. Duda, R.O., Hart, P.E.: Use of the Hough transformation to detect lines and curves in pictures. Communications of the ACM 15(1), 11-15 (1972)

9. Duffner, S., Garcia, C.: Pixeltrack: a fast adaptive algorithm for tracking non-rigid objects. In: Computer Vision (ICCV), 2013 IEEE International Conference on. pp. 2480-2487. IEEE (2013)

10. Gall, J., Yao, A., Razavi, N., Van Gool, L., Lempitsky, V.: Hough forests for object detection, tracking, and action recognition. Pattern Analysis and Machine Intelligence, IEEE Transactions on 33(11), 2188-2202 (2011)

11. Godec, M., Roth, P.M., Bischof, H.: Hough-based tracking of non-rigid objects. Computer Vision and Image Understanding 117(10), 1245-1256 (2013)

12. Henriques, J.F., Caseiro, R., Martins, P., Batista, J.: High-speed tracking with kernelized correlation filters. IEEE Transactions on Pattern Analysis and Machine Intelligence 37(3), 583-596 (2015)

13. Hua, Y., Alahari, K., Schmid, C.: Online object tracking with proposal selection. In: Proceedings of the IEEE International Conference on Computer Vision. pp. 3092-3100 (2015)

14. Kristan, M., Leonardis, A., Matas, J., al.: The visual object tracking VOT2016 challenge results (2016)

15. Kristan, M., Matas, J., Leonardis, A., al.: The visual object tracking VOT2015 challenge results. In: Proceedings of the IEEE International Conference on Computer Vision Workshops. pp. 1-23 (2015)

16. Kristan, M., Pflugfelder, R., Leonardis, A., Matas, J., al.: The visual object tracking vot2014 challenge results (2014), http://www.votchallenge.net/vot2014/ program.html

17. Maresca, M.E., Petrosino, A.: Matrioska: A multi-level approach to fast tracking by learning. In: Image Analysis and Processing-ICIAP 2013, pp. 419-428. Springer (2013)

18. Maresca, M.E., Petrosino, A.: Clustering local motion estimates for robust and efficient object tracking. In: European Conference on Computer Vision. pp. 244253. Springer (2014)

19. Nam, H., Baek, M., Han, B.: Modeling and propagating cnns in a tree structure for visual tracking. arXiv preprint arXiv:1608.07242 (2016)

20. Nam, H., Han, B.: Learning multi-domain convolutional neural networks for visual tracking. In: Proceedings of the IEEE Conference on Computer Vision and Pattern Recognition. pp. 4293-4302 (2016)

21. Possegger, H., Mauthner, T., Bischof, H.: In defense of color-based model-free tracking. In: Proceedings of the IEEE Conference on Computer Vision and Pattern Recognition. pp. 2113-2120 (2015)

22. Vojíŕ, T., Matas, J.: The enhanced flock of trackers. In: Registration and Recognition in Images and Videos, pp. 113-136. Springer (2014)

23. Vojir, T., Noskova, J., Matas, J.: Robust scale-adaptive mean-shift for tracking. In: Scandinavian Conference on Image Analysis. pp. 652-663. Springer (2013)

24. Yang, F., Lu, H., Yang, M.H.: Robust visual tracking via multiple kernel boosting with affinity constraints. IEEE Transactions on Circuits and Systems for Video Technology 24(2), 242-254 (2014)

25. Yilmaz, A., Javed, O., Shah, M.: Object tracking: A survey. ACM computing surveys (CSUR) 38(4), 13 (2006) 